\title{
ORIGINAL ARTICLE Age-related inflammatory bone marrow microenvironment induces ineffective erythropoiesis mimicking del(5q) MDS
}

\author{
Y Mei ${ }^{1}$, B Zhao ${ }^{1}$, AA Basiorka ${ }^{2}, \mathrm{~J} \mathrm{Yang}{ }^{1}, \mathrm{~L} \mathrm{Cao}^{1,3}, \mathrm{~J} \mathrm{Zhang}^{1}, \mathrm{~A} \mathrm{List}^{2,4}$ and $\mathrm{P} \mathrm{Ji}^{1}$
}

Anemia is characteristic of myelodysplastic syndromes (MDS). The mechanisms of anemia in MDS are unclear. Using a mouse genetic approach, here we show that dual deficiency of mDia1 and miR-146a, encoded on chromosome 5q and commonly deleted in MDS (del(5q) MDS), causes an age-related anemia and ineffective erythropoiesis mimicking human MDS. We demonstrate that the ageing bone marrow microenvironment is important for the development of ineffective erythropoiesis in these mice. Damageassociated molecular pattern molecules (DAMPs), whose levels increase in ageing bone marrow, induced TNFa and IL-6 upregulation in myeloid-derived suppressor cells (MDSCs) in mDia1/miR-146a double knockout mice. Mechanistically, we reveal that pathologic levels of TNFa and IL-6 inhibit erythroid colony formation and differentially affect terminal erythropoiesis through reactive oxygen species-induced caspase-3 activation and apoptosis. Treatment of the mDia1/miR-146a double knockout mice with all-trans retinoic acid, which promoted the differentiation of MDSCs and ameliorated the inflammatory bone marrow microenvironment, significantly rescued anemia and ineffective erythropoiesis. Our study underscores the dual roles of the ageing microenvironment and genetic abnormalities in the pathogenesis of ineffective erythropoiesis in del(5q) MDS.

Leukemia (2018) 32, 1023-1033; doi:10.1038/leu.2017.326

\section{INTRODUCTION}

Myelodysplastic syndromes (MDS) are age-related bone marrow malignancies characterized by dysplastic and ineffective production of myeloid cells and risk of developing acute myeloid leukemia (AML). Clinically, a great majority of patients with MDS have anemia caused by ineffective production of erythroid cells at different stages of erythropoiesis. The pathogenesis of ineffective erythropoiesis in MDS is unclear, which can be attributed to the heterogeneous nature of many genetic and molecular abnormalities involved in the development of MDS as well as microenvironmental factors. One of the most common cytogenetic defects in MDS is the heterozygous interstitial deletion of chromosome $5 q(\operatorname{del}(5 q))$. There are two common deleted regions (CDRs) identified on 5q: a distal locus that is often deleted in $5 q$ - syndrome with good prognosis and a proximal locus deleted in patients with higher risk of MDS., ${ }^{1,2}$ Refractory anemia is a characteristic feature of $\operatorname{del}(5 q)$ MDS. $^{3}$ Recent studies have shown that haploinsufficiency of Rps 14 on the distal locus of the CDRs blocks erythroid differentiation through upregulation of p53 and its downstream genes S100a8 and S100a9. ${ }^{4}$ Whether deficiencies of other genes on chromosome $5 q$, and the ageing bone marrow microenvironment, are involved in ineffective erythropoiesis is unknown.

Growing evidence reveals that deregulation of innate immune responses is also involved in the pathogenesis of $\operatorname{del}(5 q)$ MDS. $^{5-11}$ Concurrent loss of miR-145 and miR-146a, which are located at the distal region of $5 q$, leads to the dysplastic phenotype in megakaryocytes by the upregulation of their downstream target tumor necrosis factor receptor-associated factor-6 (TRAF6). ${ }^{11}$
Subsequent studies using miR-146a knockout mice show that miR-146a serves as a brake on inflammation and regulates myeloproliferation and oncogenic transformation. ${ }^{12}$ Our previous work shows that mDia1, whose encoding gene is flanked by the two CDRs on $5 q$, is significantly decreased in del(5q) MDS CD34 ${ }^{+}$ cells. mDia1 heterozygous and knockout mice developed agerelated neutropenia and myeloid dysplasia mimicking human MDS. ${ }^{5}$ Mechanistically, CD14 is aberrantly overexpressed on granulocytes, leading to a hypersensitive innate immune response to lipopolysaccharide (LPS) stimuli. A more recent study illustrates that loss of another $5 q$ gene, Tifab, alters hematopoiesis through derepression of Toll-like receptor-TRAF6 pathway, leading to ineffective hematopoiesis and cytopenia. ${ }^{7}$ In these studies, leukopenia, including neutropenia, is commonly observed, which demonstrates that deregulation of the innate immune signaling is involved in myeloid dysplasia. However, anemia is mild or not observed in these models, indicating that either loss of these genes are not essential for the development of ineffective erythropoiesis, or combined deficiencies, as those in $\operatorname{del}(5 q)$ MDS, are required for the clinical manifestation of anemia.

In this study, we generate a mouse model with concurrent deletion of mDia1 and miR-146a, both are encoded by genes on chromosome $5 q$ and involved in the repression of TLR-TRAF6 pathway. Mice with mDia1/miR-146a double knockout develop age-related anemia and ineffective erythropoiesis. Our study illustrates the importance of the age-related inflammatory bone marrow microenvironment in the pathogenesis of ineffective erythropoiesis and anemia in $\operatorname{del}(5 q)$ MDS.

\footnotetext{
${ }^{1}$ Department of Pathology, Feinberg School of Medicine, Northwestern University, Chicago, IL, USA; ${ }^{2}$ Cancer Biology PhD Program, H. Lee Moffitt Cancer Center and Research Institute and the University of South Florida, Tampa, FL, USA; ${ }^{3}$ Department of Hematology and Oncology, Children's Hospital of Soochow University, Suzhou, China and

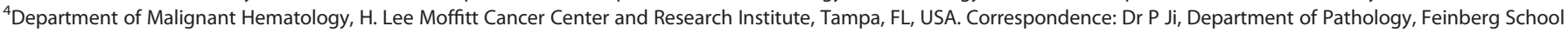
of Medicine, Northwestern University, 303 East Chicago Avenue, Ward 3-210, Chicago, IL 60611, USA.

E-mail: peng-ji@fsm.northwestern.edu

Received 6 July 2017; revised 22 October 2017; accepted 26 October 2017; accepted article preview online 16 November 2017 ; advance online publication, 5 January 2018
} 


\section{MATERIALS AND METHODS}

Experimental methods are in the supplementary materials.

\section{RESULTS}

Dual deficiency of mDia1 and miR-146a causes age-related anemia in mice.

To characterize the extent of pathologically activated innate immune responses on the development of anemia in del(5q) MDS, we crossed the mDia1 knockout mice with miR-146a knockout mice and generated mDia1/miR-146a double knockout (hereafter DKO) mice. We monitored the complete blood counts in these mice over 1 year. mDia1 KO mice showed no anemia and miR-146a KO mice had mild anemia compared to the DWT control mice. In comparison, the DKO mice developed significant agerelated anemia starting at 7 months, which became worse with age (Figure 1a). The DKO mice also displayed thrombocytopenia compared to single KO and DWT control mice. Both DKO and
mDia1 single KO mice developed neutropenia (Figure 1a), which is consistent with previous findings. ${ }^{5}$ Morphologic examination of the peripheral blood in the aged DKO mice showed severe anemia with anisopoikilocytosis including hypochromatic cells, HowellJolly bodies, and increased reticulocyte count (Supplementary Figures $1 a$ and $b$ ).

We next examined the bone marrow of the 1-year old mice to determine the cause of anemia. Gross observation showed pale bone and bone marrow cell aspirate from DKO mice and to a lesser extent from miR-146a knockout mice (Supplementary Figure $1 \mathrm{c}$ ). We reasoned that the pale appearance of the DKO bone marrow was due to the decreased erythroid population. Accordingly, total bone marrow cells and Ter119 (a maturing erythroid marker) positive erythroid cells were significantly decreased in DKO mice compared to wild type and single KO littermate controls, confirming our hypothesis (Figures $1 \mathrm{~b}$ and $\mathrm{c}$ ). The DKO mice also exhibited increased lethality around 1-year old, likely due to a life-threatening anemia (Figure 1d).

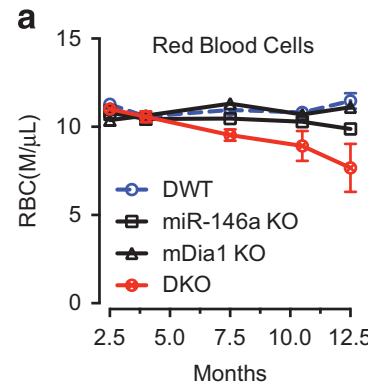

b

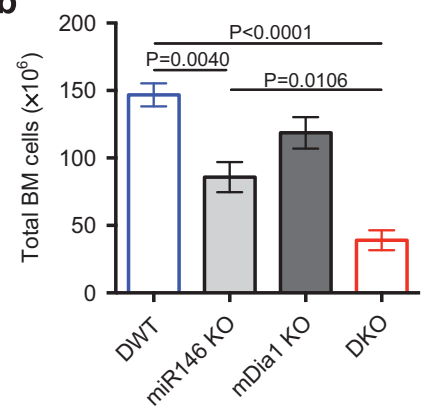

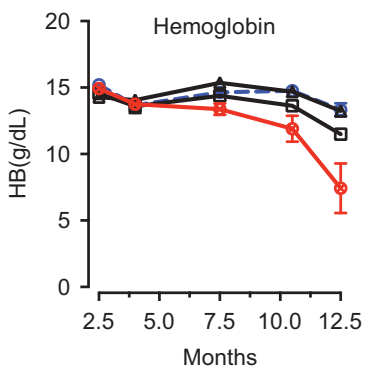

C

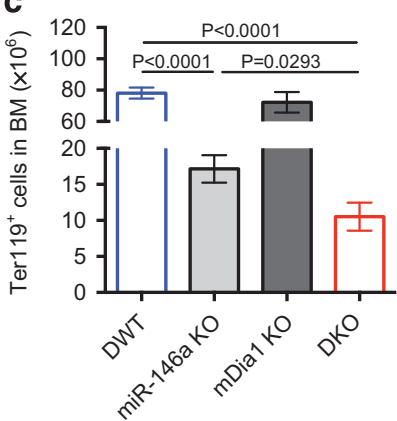

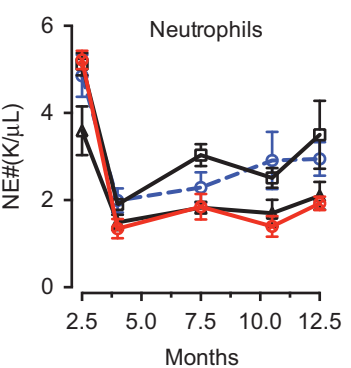

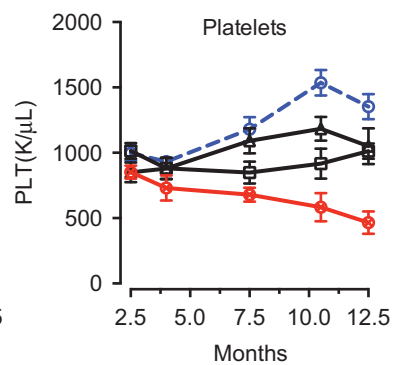

d

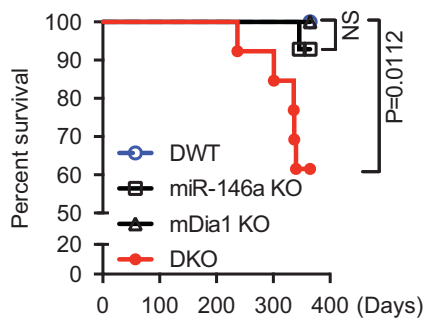

e

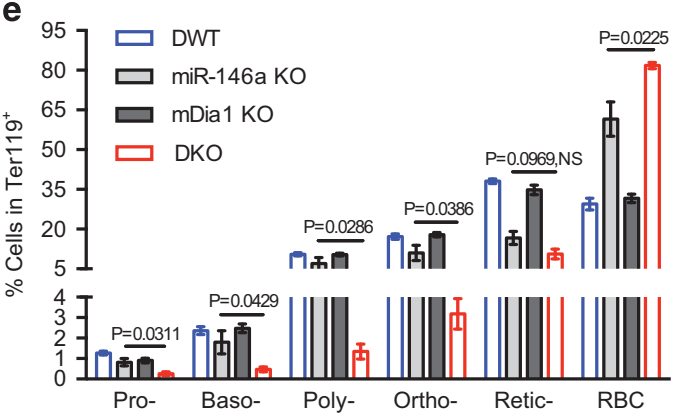

f

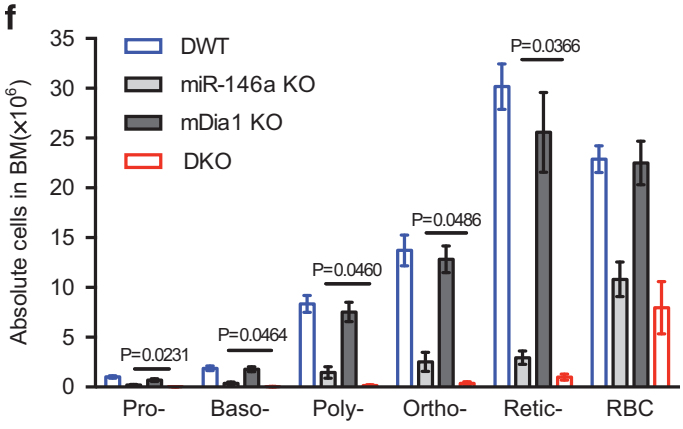

Figure 1. mDia1/miR-146a double knockout mice develop age-related anemia and ineffective erythropoiesis. (a) Complete blood count of different time points of mDia1/miR-146a double wild type (DWT), mDia1 or miR-146a single knockout (KO), and double knockout (DKO) mice. DWT, $n=6$; miR-146a KO, $n=6$; mDia1 KO, $n=5$; DKO, $n=7$. (b, c) Flow cytometric analysis of bone marrow cells from indicated mice at 1 year old. Total bone marrow cells (b) and absolute Ter119 ${ }^{+}$erythroid cells (c) from femur and tibia were presented. DWT, $n=6 ; \mathrm{miR}-146 \mathrm{~K}$ KO, $n=4$; mDia1 KO, $n=5$; DKO, $n=5$. (d) Kaplan-Meier survival analysis of the indicated mice. DWT, $n=14 ;$ miR-146a KO, $n=8 ; \mathrm{mDia} 1 \mathrm{KO}, n=6$; DKO, $n=11$. Both males and females were included. (e, f) The Ter $119^{+}$erythroid cells in c were analyzed by CD44 levels and forward scatter to define various developmental stages of erythroblasts (Pro-: proerythroblasts, Baso-: basophilic erythroblasts, Poly-: polychromatic erythroblasts, Ortho-: orthochromatic erythroblasts, reticulocytes (Retic-) and mature red blood cells (RBC)). Quantification of the percentages and absolute cell numbers of erythroblasts at each stage of differentiation were presented in e and f, respectively. DWT, $n=6 ;$ miR-146a KO, $n=4$; mDia1 KO, $n=5$; DKO, $n=5$. 
To further dissect the aberrancies in erythroid cell differentiation, we next performed a flow cytometric analysis using CD44 and forward scatter to divide the erythroid cells into different developmental stages. ${ }^{13}$ The percentage of nucleated erythroblasts in the aged (>1-year old) DKO bone marrow was significantly decreased compared to littermate controls (Figure 1e and Supplementary Figure 1d). miR-146a KO mice also showed slightly decreased percentage of nucleated erythroblasts (Figure 1e and Supplementary Figure 1d). When the absolute number of erythroblasts was calculated from leg bones, aged miR-146a knockout mice showed a significant decrease, and erythroblasts from aged DKO mice further decreased compared to miR-146a knockout mice (Figure 1f).

Unlike patients with MDS, mice with anemia often show compensatory erythropoiesis evidenced by splenomegaly. ${ }^{14}$ As expected, the old DKO mouse showed a dramatically increased spleen size compared to wild type or single knockout littermate controls (Supplementary Figures $2 \mathrm{a}$ and $\mathrm{b}$ ). The percentage of splenic Ter119-positive erythroid cells increased by $20-30 \%$ in DKO mice due to stress erythropoiesis. ${ }^{15-17}$ The percentages of lymphocytes were significantly reduced (Supplementary Figure 2c). We next examined different stages of terminal erythropoiesis in the spleen. Unlike the bone marrow, terminal erythropoiesis in the spleen did not show defects in various stages of differentiation (Supplementary Figure 2d), These data suggest that ineffective erythropoiesis in the bone marrow is compensated by splenic stress erythropoiesis, likely protecting the DKO mice from early death induced by severe anemia. We further demonstrated that the compensatory splenic stress erythropoiesis is similarly age-related (Supplementary Figures 2e-h).

Age-related bone marrow microenvironment contributes to the development of anemia.

Both mDia1 and miR-146a knockout mice show age-related increased secretion of proinflammatory cytokines, mostly TNF-a and IL-6. ${ }^{5,11,12}$ To determine whether the defects of erythroid population are erythroid cell intrinsic or due to the inflammatory microenvironment, we performed two groups of transplantation experiments. In these experiments, bone marrow mononuclear cells from aged (>1-year old) DKO mice or age-matched DWT littermate controls were transplanted into either young (2-month old) or aged ( $>1$-year old) recipient mice (Figure 2). Like the nontransplanted aged DKO mice, these transplanted mice exhibited neutropenia early post-transplantation due to the cell-intrinsic effect of mDia1 deficiency, as well as worsening thrombocytopenia (Figure $2 b$ ). The young recipient mice showed no significant differences in red cell indices between DKO and DWT transplanted mice until 6-month post-transplantation when RBC count, hemoglobin, and hematocrit began declining (Figure 2c). The age when anemia manifested in these transplanted mice was similar to that of the regular DKO mice (Figure 1a), implicating an age-related microenvironment in the development of anemia. a

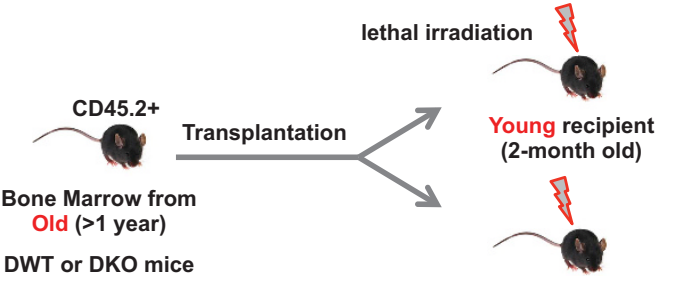

Old recipient (>1-year old)

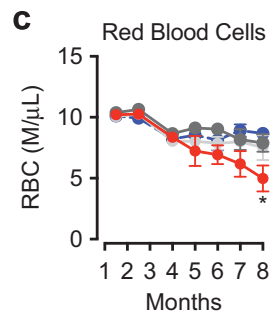

e

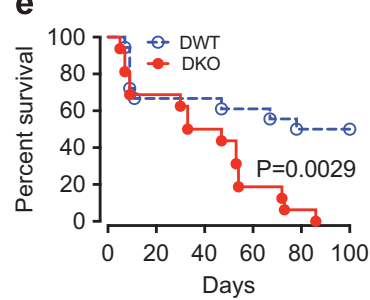

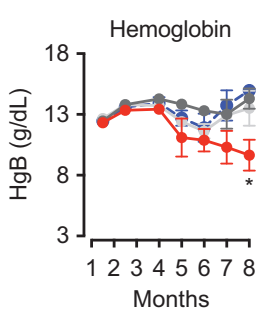
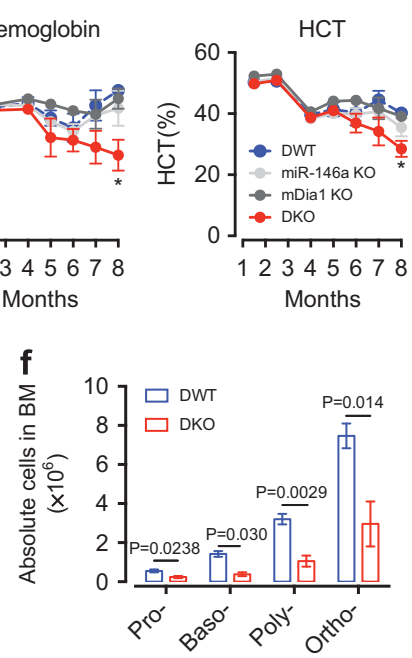

b
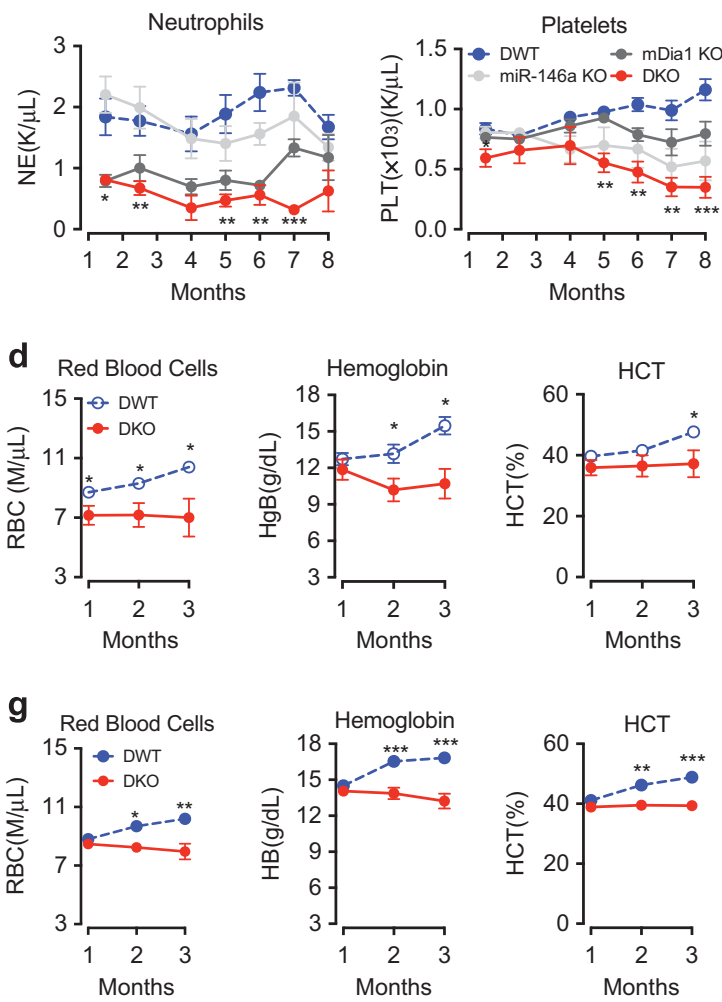

Figure 2. The aging bone marrow microenvironment plays an important role in the development of anemia and ineffective erythropoiesis. (a) Schematic overview of the bone marrow transplantation experiments. (b, c) Post-transplant complete blood counts at the indicated time of recipient mice (2-month old when transplanted) transplanted with bone marrow cells from old DWT, mDia1 KO, miR-146a KO, or DKO mice ( $>$ 1-year-old). $N=5$ in each group. (d) Post-transplant red cell indices at the indicated time of recipient mice (> 1-year-old when transplanted) transplanted with bone marrow cells from old DWT or DKO mice ( $>1$-year-old). $N=10$ in each group. (e) Kaplan-Meier survival analysis of the recipient mice in (d). $N=18$ in DWT; $N=16$ in DKO. (f) The bone marrow Ter $119^{+}$erythroid cells from both sets of transplantation experiments were analyzed by CD44 levels and forward scatter to define various developmental stages of erythroblasts as in Figure $1 \mathrm{f}$. $N=3$ in each group. (g) Post-transplant red cell indices at the indicated time of the recipient mice ( $>$ 1-year-old when transplanted) transplanted with bone marrow cells from young DWT or DKO mice (2-month-old). $N=9$ in DWT; $N=7$ in DKO. 
Old ( $>1-$ year of age) recipient mice transplanted with old DWT or DKO bone marrow cells showed initial anemia due to their age. Those transplanted with DWT bone marrow cells gradually recovered from transplantation stress. However, the old recipient mice transplanted with old DKO bone marrow cells exhibited persistent anemia starting from one month post-transplantation (Figure $2 \mathrm{~d}$ ). The old recipient mice transplanted with old DKO bone marrow cells also exhibited rapid lethality compared to the old DWT bone marrow transplanted controls (Figure 2). When we analyzed the bone marrow of the old recipient mice from both groups of the transplantation experiments, we found ineffective erythropoiesis at different stages of red cell development similar to the non-transplanted old DKO mice (Figure 2f). Furthermore, when we transplanted young DKO bone marrow cells into old recipient mice (>1-year of age), the recipient mice failed to recover from anemia compared to DWT transplanted ones (Figure 2g). Taken together, these results indicate that the agerelated bone marrow microenvironment plays an important role in the development of ineffective erythropoiesis and anemia.

Proinflammatory cytokines are over-produced in the bone marrow myeloid-derived suppressor cells in aged mDia1/miR-146a double knockout mice.

With the dramatic decrease of the erythroid population in the bone marrow of the aged DKO mice, the relative percentage of Gr1 and Mac1 double-positive myeloid cells was significantly increased (Figure $3 a$ and b). The absolute number of myeloid cells was decreased in the bone marrow of DKO mice, albeit to a lesser extent than the decrease observed in the erythroid population (Supplementary Figure 3a). Consistent with the flow cytometric results, morphologic examination of the bone marrow from the old DKO mice showed marked erythroid hypoplasia with most of the cells in the myeloid lineage (Figure 3). Many of these myeloid cells were immature appearing with large cell size measured by forward scatter by flow cytometry (Figure 3d and e). However, the DKO mice did not develop acute leukemia demonstrated by their decreased numbers of lineage negative cells as well as stem and progenitor cells (Supplementary Figures 3b-f). Megakaryocytes were markedly decreased in the bone marrow with compensatory increased in the spleen in DKO mice (Supplementary Figures 3g,h), consistent with thrombocytopenia in these mice.

A previous report demonstrated that myeloid-derived suppressor cells (MDSCs), which are characteristically Gr1 and Mac1 double-positive, immature appearing, and immunosuppressive, are enriched in the bone marrow in MDS patients. ${ }^{18}$ Indeed, the immature-appearing myeloid cells in the old DKO mice fulfill these criteria and blocked T cell activation when co-cultured with CD4 positive $\mathrm{T}$ cells in vitro (data not shown). Consistently, MDSCs, including the monocytic-MDSCs that were reported to have stronger suppressive functions, ${ }^{19}$ were significantly increased in the peripheral blood of DKO mice. Consequently, the lymphoid population was significantly decreased (Supplementary Figure 3i).

The ageing bone marrow microenvironment is composed of increasing amounts of damage-associated molecular patterns (DAMPs), which are potent inducers of proinflammatory cytokines production, especially in cells with sensitized innate immune signaling. 8,9,20 To analyze whether treatment of DAMPs could induce the overproduction of proinflammatory cytokines in the MDSCs in the DKO mice in vitro, we purified Gr1 and Mac1 doublepositive cells from the bone marrow of young DKO mice and their littermate controls. These cells were treated with DAMPs prepared through repetitive freeze and thaw cycles of wild-type bone marrow cells. ${ }^{20,21}$ Indeed, treatment with DAMPs induced overproduction of TNF- $a$ and IL- 6 in the bone marrow-derived myeloid cells from each group of mice, with levels much higher in the MDSCs from DKO cells (Figure 3f). Particularly, IL-6 presented the most profound (up to 200 -fold change) upregulations, suggesting a more crucial role of IL-6 in the pathogenesis in DKO mice.

We next analyzed the serum levels of inflammatory cytokines using a mouse cytokine/chemokine panel. As expected, the serum levels of IL- 6 and TNFa were significantly increased in old DKO mice compared to age-matched DWT and single knockout controls (Supplementary Figure 4), which was further confirmed by conventional ELISA assay (Figure $3 \mathrm{~g}$ ). The level of IL-10, which is highly expressed by MDSCs, ${ }^{22}$ was also significantly upregulated in DKO mice (Supplementary Figure 4), consisting with the increased MDSCs in DKO mice. These results collectively indicate that bone marrow microenvironment-mediated ineffective erythropoiesis in DKO mice could be related to the over-secretion of proinflammatory cytokines.

TNFa and IL-6 negatively affect erythropoiesis through different mechanisms.

Proinflammatory cytokines are known to be involved in anemia of chronic diseases (ACD) through upregulation of liver hepcidin, which limits the availability of iron to the developing erythroblasts. ${ }^{23-25}$ However, the increased cytokine levels did not induce hepcidin upregulation in hepatocytes from aged DKO mice compared to the control mice (Figure 4a), indicating that ineffective erythropoiesis in aged DKO mice was likely hepcidinindependent. Next, we investigated whether the proinflammatory cytokines TNFa and IL- 6 directly affect erythropoiesis in vitro. We first purified the lineage negative bone marrow cells from old ( $>$ 1 year of age) DWT and DKO mice and cultured them in erythropoietin-containing medium for three days. No statistically significant differences were observed in their differentiation, as quantified by CD71 and Ter119 double-positive cells (Figure 4b). Similar findings were obtained from bone marrow lineage negative cells of young and old DKO mice, mDia1 or miR-146a single knockout mice, and various other bone marrow progenitor cells cultured in vitro (data not shown and Supplementary Figures $5 a, b)$, which further supports that ineffective erythropoiesis is erythroid cell non-autonomous. Therefore, we used DWT bone marrow cells to determine how proinflammatory cytokines affect various stages of erythropoiesis. Previous studies have shown inhibitory effects of proinflammatory cytokines in erythropoiesis. ${ }^{20}$ However, the mechanisms of cytokine-mediated inhibition of erythropoiesis, the stages at which the inhibitory effects are conferred, and whether there are any differences between TNFa and IL-6 in inducing ineffective erythropoiesis, are unknown.

To answer these questions, we purified bone marrow cells from young DWT bone marrow and cultured the cells in methocellulose with erythropoietin and increasing amounts of TNFa and IL-6. Both TNFa and IL-6 induced a dose-dependent decrease in Colony-Forming Unit- Erythroid (CFU-E) colony formation (Figure 4c), demonstrating that increasing levels of both cytokines affect the early stage of erythropoiesis. After the CFU-E stage, the erythroid cells undergo terminal erythropoiesis that can be monitored by flow cytometry. We next determined the differentiation of DWT bone marrow lineage negative cells cultured with erythropoietin and increasing amount of TNFa and IL-6. These cytokines slightly inhibited cell differentiation on days 2 and 3 , as measured by Ter119-positive cells. However, both TNFa and IL-6 induced increased Ter119-positive cells on day 1 (Supplementary Figure 5c), possibly contributing to the depletion of erythroid progenitor cells.

We next found that IL- 6 induced a dose-dependent increase in annexin $\mathrm{V}$ positive apoptotic cells at different stages of terminal erythropoiesis. TNFa-mediated apoptosis (annexin V positive) was only observed on day 3 (Figure 4d). TNFa also induced mild necrosis (annexin $\mathrm{V}$ negative and PI positive) on day 2 and 3 whereas IL-6-treated cells showed no increased necrosis compared to the vehicle controls (Supplementary Figure $5 \mathrm{~d}$ ). 

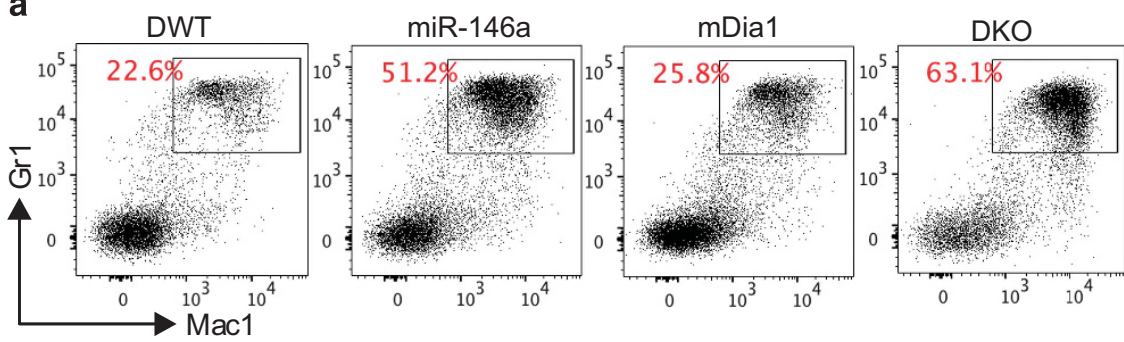

C
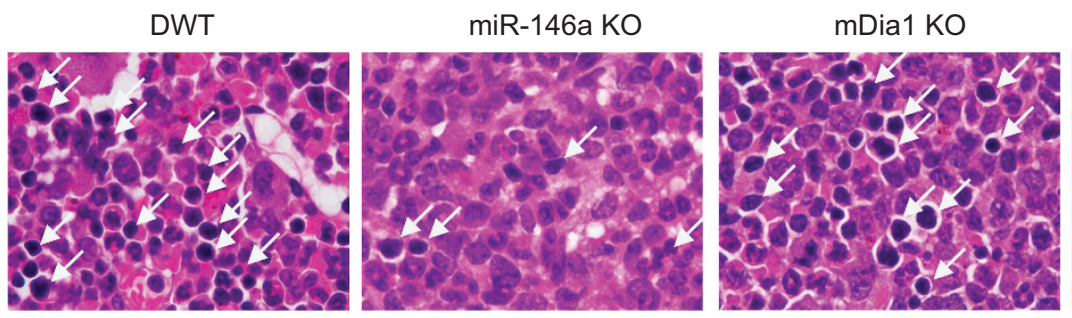

$\mathrm{mDia1} \mathrm{KO}$
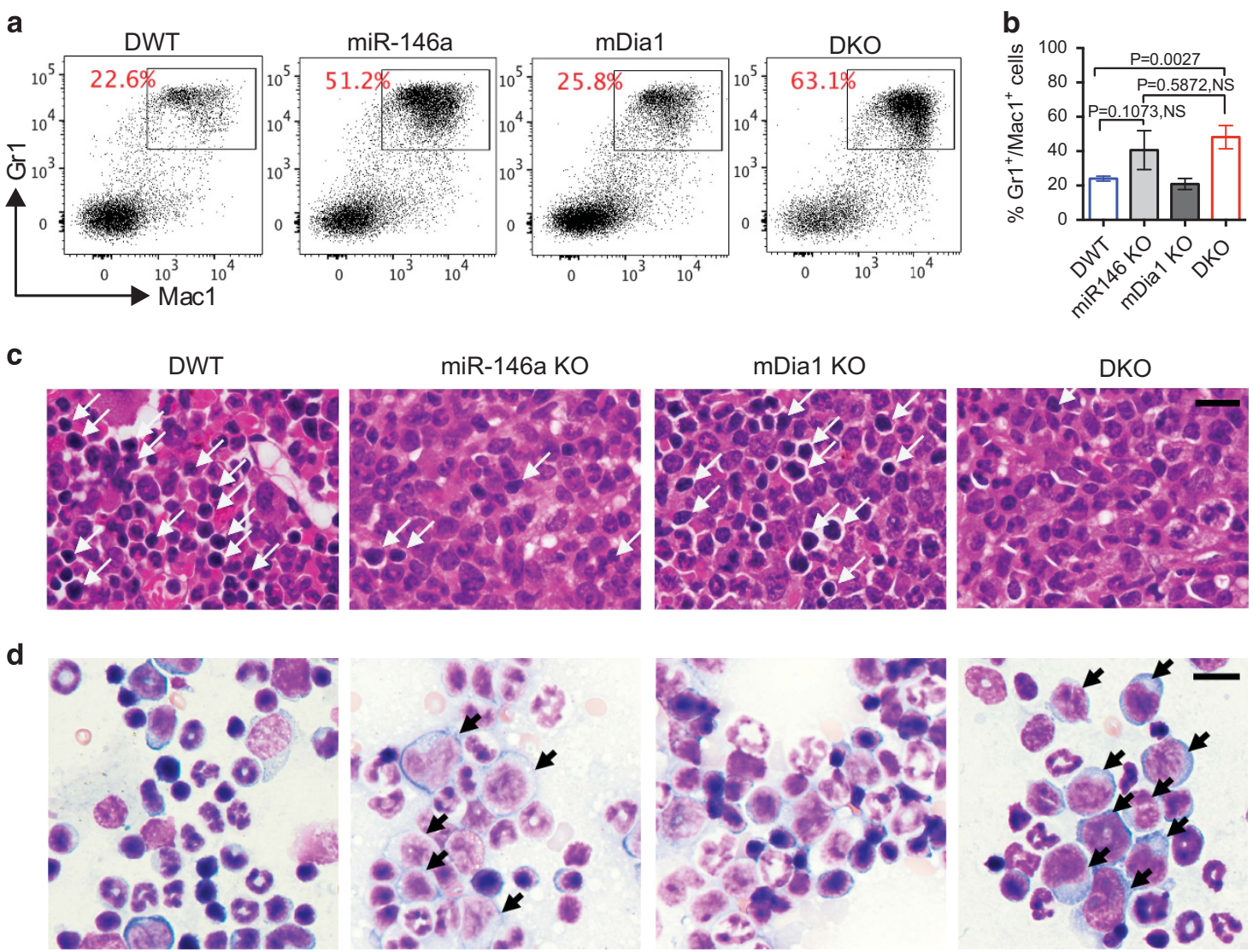

e
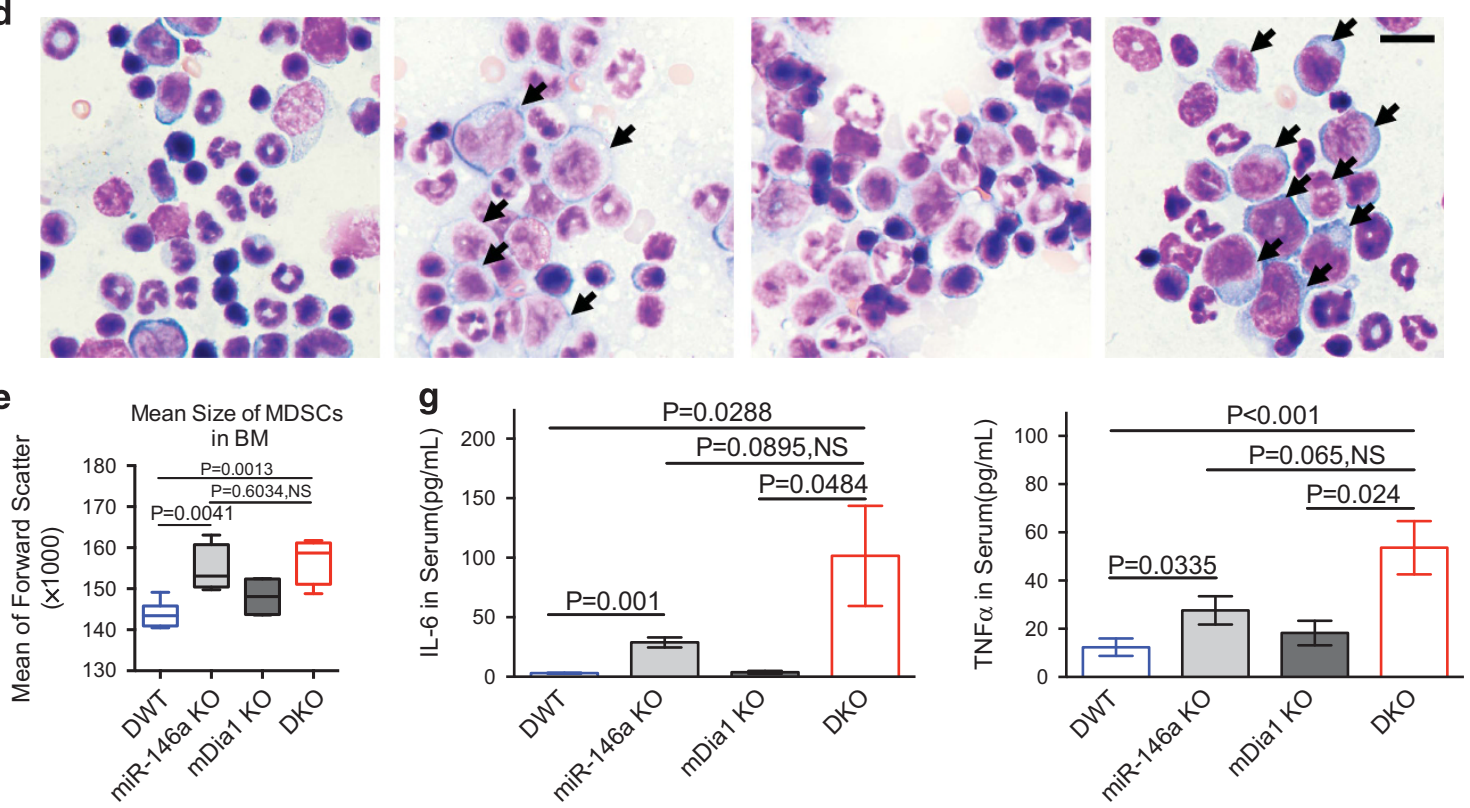

f

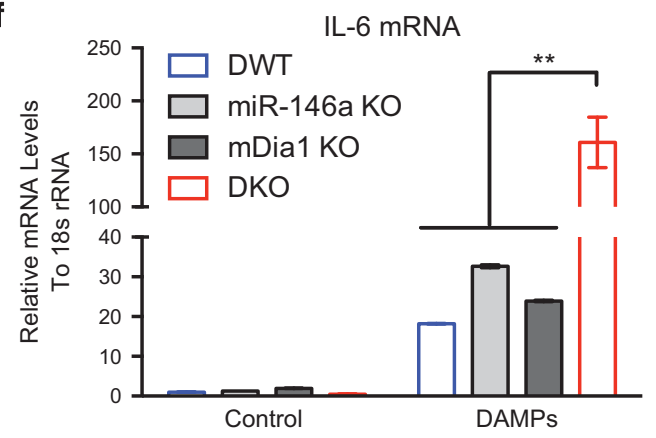

Figure 3. Proinflammatory cytokines are over-produced by myeloid-derived suppressive cells in aged mDia1/miR-146a double knockout mice. (a, b) Flow cytometric analysis of Mac1 and Gr1 positive cells in the bone marrow from indicated mice at 1 year old. The percentages of Gr1/ Mac1 double-positive cells from femur and tibia were presented in b. DWT, $n=6$; miR-146a KO, $n=4 ; \mathrm{mDia} 1 \mathrm{KO}, n=5 ; \mathrm{DKO}, n=5$. (c) $\mathrm{H} \& \mathrm{E}$ stains of bone marrow sections of indicated mice. Arrows indicate erythroblasts. Scale bar: $50 \mu \mathrm{m}$. (d) Bone marrow smear prepared from the indicated mice and stained with Wright-Giemsa. Black arrows indicate immature-appearing myelocytes. Scale bar: $30 \mu \mathrm{m}$. (e) Statistical analysis of the size of immature-appearing MDSCs measured by flow cytometry forward scatter of Gr1/Mac1 double-positive cells from indicated mice. Data were presented as min- to max-box and whisker plots. (f) Gr1/Mac1 double-positive granulocytes from the indicated mice were purified and challenged with damage-associated molecular pattern proteins (DAMPs) (1:20 for $2 \mathrm{~h}$ ). The relative mRNA levels of TNF $\alpha$ and IL- 6 were determinate by real-time PCR analysis. (g) Serum IL-6 and TNF $\alpha$ from indicated mice ( $>$ 1-year-old) were assayed by ELISA. DWT, $n=6$; miR-146a KO, $n=4$; mDia1 KO, $n=5$; DKO, $n=6$. 

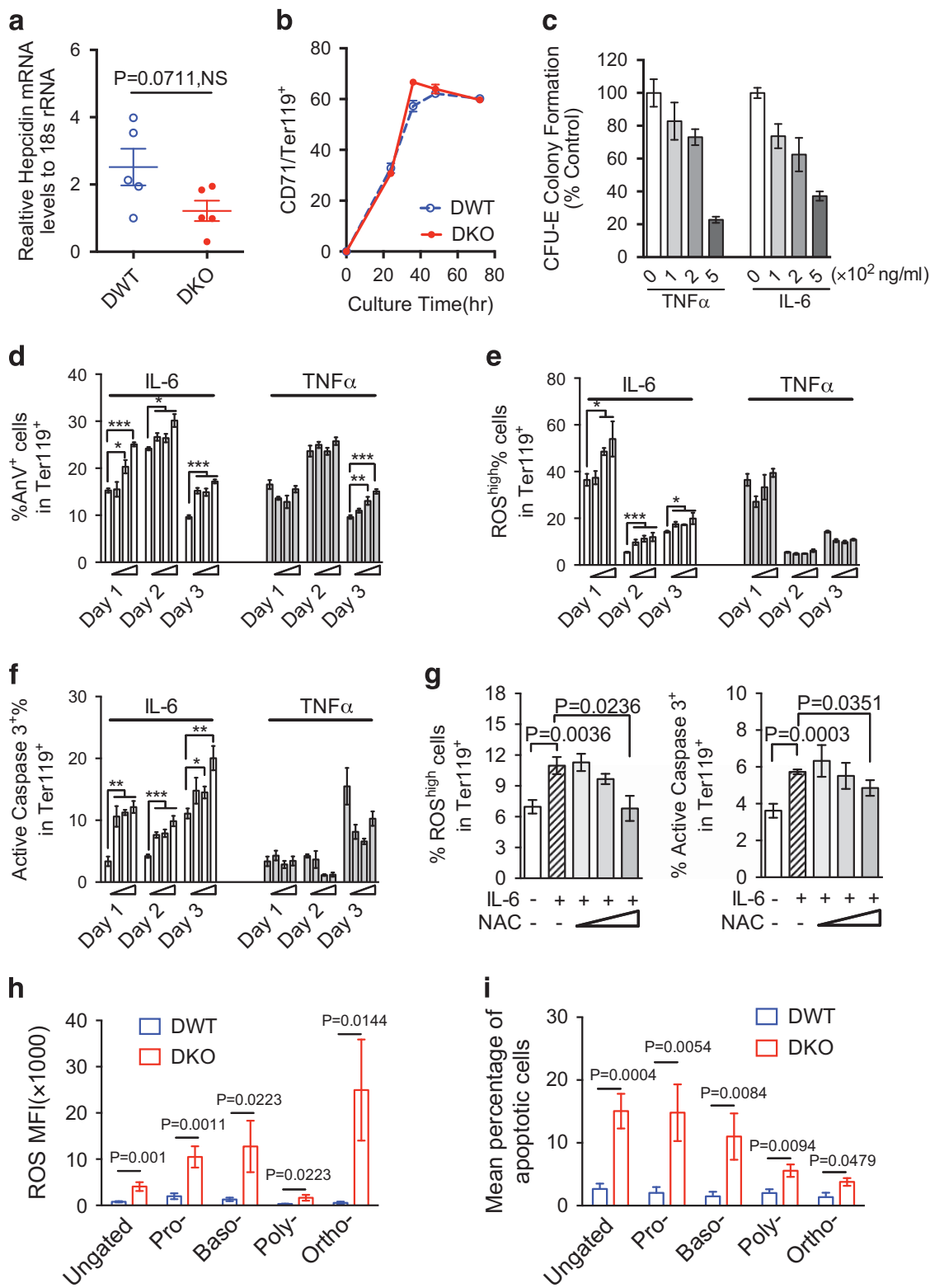

Figure 4. IL-6 and TNF $\alpha$ induce ineffective erythropoiesis in vitro through distinct mechanisms. (a) Quantification of hepcidin mRNA levels in hepatocytes by real-time PCR. $N=5$ in each group. (b) Bone marrow lineage negative cells (Lin ${ }^{-}$) from indicated mice were cultured in erythroid differentiation medium containing $2 \mathrm{U} / \mathrm{ml}$ erythropoietin. Cell differentiation was analyzed by flow cytometric analysis comparing the expression of transferrin receptor CD71 and Ter119. (c) CFU-E colony formation assay of bone marrow cells cultured in methylcellulose medium with $3 \mathrm{U} / \mathrm{ml}$ erythropoietin and increasing amounts of TNF $\alpha$ and IL-6. (d) Lin cells from wild-type mice were purified and cultured in erythroid differentiation medium in the presence of increasing amounts of IL- 6 and TNF $\alpha(50,100$, and $200 \mathrm{ng} / \mathrm{ml})$ for 3 days. The annexin V positive cells in the Ter $119^{+}$erythroid cells were quantified. (e, f) The ROS and active caspase-3 levels were assayed by flow cytometry in Ter119-positive cells from (d), and were shown in e and f, respectively. (g) Lin cells were pre-treated with $100 \mathrm{ng} / \mathrm{ml} \mathrm{IL-6} \mathrm{in} \mathrm{EPO} \mathrm{medium} \mathrm{for}$ $24 \mathrm{~h} .1-20 \mu \mathrm{M}$ NAC were then added directly into the medium and the cells were cultured for another $24 \mathrm{~h}$. The ROS levels and active caspase3 in the cells were assayed by flow cytometric analysis. Relative levels of ROS and active caspase-3 levels were presented. All the data are shown as mean \pm s.e.m. and representative data from 3 independent experiments. NS: not significant. ${ }^{*} P<0.05 ;{ }^{* *} P<0.01 ;{ }^{* * *} P<0.001$. (h) Mean fluorescence intensity (MFI) of ROS positive cells were analyzed at different populations of the erythroblasts and ungated bone marrow erythroid populations in old mDia1/miR-146a DWT or DKO mice ( $>1$-year-old). DWT, $n=8$; DKO, $n=5$. (i) Mean percentages of apoptotic cells in different populations and ungated bone marrow erythroblasts from indicated mice ( $>1$-year-old). DWT, $n=7 ; \mathrm{DKO}, n=4$.

Pathologic levels of both IL-6 and TNFa induced downregulation of GATA1 (Supplementary Figures 5e and f), a critical transcription factor in erythropoiesis. ${ }^{26}$ TNFa caused the most significant downregulation of GATA1 from the mRNA level (Supplementary Figure $5 \mathrm{~g}$ ), which further reduced the expression of the downstream targets of GATA1, including hemoglobin and Fog-1 (data not shown).
Reactive oxygen species (ROS) induced by proinflammatory cytokines are known to promote apoptosis. ${ }^{27}$ Our previous report demonstrated that ROS presents at the highest level in the early stages of terminal erythropoiesis, which corresponds to day 1 of the erythroid differentiation culture system. ${ }^{28}$ ROS levels gradually decreased on day 2 and 3 in untreated cells (Figure 4e). Administration of IL- 6 led to a dose-dependent increase of ROS 
beyond the physiologic level, which was most prominent on day 1. In contrast, TNFa-treated cells did not show increased ROS levels (Figure 4e). Consistent with the role of ROS in the activation of caspase-3, IL-6-treated cells exhibited a dose-dependent increase of active caspase-3, correlating with their apoptotic cell death. No increased activation of caspase-3 was observed in TNFatreated cells (Figure 4f). Cell cycle analysis showed that the percentage of $S$ and G2/M phases were slightly increased with the treatment of IL-6 and TNFa on day 1 (Supplementary Figure 5h), which could contribute to the increased percentage of Ter $119^{+}$ cells (Supplementary Figure $5 c$ ).

To further confirm the role of IL-6-induced ROS in the activation of caspase-3, we treated the IL-6 $(100 \mathrm{ng} / \mathrm{ml})$ challenged bone marrow lineage negative progenitor cells with increasing amount of ROS scavenger, $\mathrm{N}$-acetyl-cysteine (NAC). Indeed, NAC dosedependently rescued the increased ROS formation and activation of caspase-3 when the lineage negative cells underwent differentiation to erythroid cells (Figure $4 \mathrm{~g}$ ). Taken together, these results demonstrate that TNFa and IL-6 negatively affect erythropoiesis through different mechanisms. Both cytokines compromise CFU-E colony formation at higher levels. In addition, IL-6 also induces ROS-mediated activation of caspase- 3 and apoptosis, which is not observed with TNFa.

We next determine whether ROS and apoptosis were increased in vivo in erythroid cells from DKO mice. Like the in vitro cytokinetreated erythroid cells, ROS levels were markedly elevated beyond the physiologic level at different stages of terminal erythropoiesis in DKO mice compared to DWT controls (Figure 4h). Annexin V positive apoptotic cells were also significantly increased in the Ter119-positive erythroid cells from DKO mice compared to DWT or single knockouts (Supplementary Figures $5 \mathrm{i}$ and $\mathrm{j}$ ). As expected, different stages of terminal erythroblasts showed increased apoptotic cells, as measured by activation of caspase-3 (Figure 4i).

Treatment of all-trans retinoic acid rescues anemia and ineffective erythropoiesis through amelioration of MDSC-induced bone marrow inflammatory microenvironment in DKO mice

Having demonstrated that the Gr1/Mac1 double-positive MDSCs secreted a large amount of TNFa and IL-6, we ask whether reducing the amount of immature MDSCs through the promotion of their differentiation would rescue anemia and ineffective erythropoiesis. To this end, we treated the old ( $>1$ year of age) DKO mice with all-trans retinoic acid (ATRA), which induces the differentiation of immature myeloid cells, ${ }^{29,30}$ for three consecutive doses. Complete blood count was performed after the treatment, which showed significant rescue of anemia (Figure 5a). ATRA also significantly reverted the ineffective erythropoiesis in the bone marrow demonstrated by the recovered Ter119-positive cells in percentage and total number (Figures $5 \mathrm{~b}$ and $\mathrm{c}$ ).

We further analyzed the ATRA-treated DKO mice and found that serum TNFa and IL- 6 was significantly decreased (Figure $5 \mathrm{~d}$ ). The mRNA levels of TNFa and IL- 6 were also markedly decreased almost to the control level in the DKO $\mathrm{Gr}^{+} / \mathrm{Mac}^{+}$MDSCs treated with ATRA after DAMP stimulation in vitro (Figure 5e). Consistent with the decreased proinflammatory cytokines, the apoptotic erythroid cells were also dramatically reduced in ATRA-treated DKO mice (Figure 5f). Indeed, ATRA not only rescued ineffective erythropoiesis in DKO mice, it also reverted the T cell inhibitory effects by MDSCs (Figure $5 \mathrm{~g}$ ). Bone marrow analysis showed that the mean size of MDSCs, including both granulocytic and monocytic-MDSCs, were reduced. The percentage of more suppressive monocytic-MDSCs was also reduced, which is accompanied by the increased percentage of granulocytic MDSCs (Figure 5h). In addition, we found that when we treated the DKO mice with ATRA beginning at 5-month old when they still did not show obvious MDS phenotypes, these mice remained free of anemia around 7-month old. In contrast, the control DKO group already showed obvious decreases of hemoglobin and hematocrit (Supplementary Figure 6). These data indicate that ATRA also plays a preventive effect in the development of anemia in DKO mice.

In vitro IL-6-treated bone marrow erythroblasts share common gene expression profiles as the erythroblasts from DKO mice To further elucidate the inhibitory effects of proinflammatory cytokine in the ineffective erythropoiesis in DKO mice, we performed microarray analyses of IL-6-challenged erythroblasts cultured in vitro as well as primary bone marrow erythroblasts at different developmental stages sorted from DWT and DKO mice. Compared to the controls, IL-6-treated erythroblasts on day 1 revealed fewer up- or downregulated genes. However, IL-6-treated erythroblasts on day 2 showed more robust changes in gene expression up to 577 significant transcripts (Figure 6a). In the erythroblasts from DKO bone marrow, 409, 518, and 474 genes were found to be changed significantly in stages I, II+III, and IV, respectively $(\mathrm{I}=$ pro-erythroblasts, $\mathrm{II}+\mathrm{III}=$ Basophilic and polychromatic erythroblasts, IV=Orthochromatic erythroblasts). DIAPH1 (encoding mDia1) and CD14 were down and upregulated respectively at all stages, which confirms the reliability of our microarray platforms (Figure 6b).

The commonly upregulated genes shared among IL- 6 treated and DKO erythroblast groups were identified and illustrated with a Venn diagram (Figure $6 \mathrm{c}$ ). These genes were applied to a clustering analysis (Supplementary Figure 7). Consistent with the induction of apoptosis in IL-6 treated and DKO erythroblasts, genes involved in the apoptotic pathway were commonly upregulated. We also found that the $\mathrm{S} 100$ proteins, including S100a4, S100a6 and S100a8, were also significantly increased (pointed out in Figure $6 a$ and b).

To comprehensively analyze the signaling pathways involved in the upregulated genes, we performed a functional PANTHER Pathway analysis. ${ }^{31}$ All five groups shared the same inflammation pathway mediated by chemokine and cytokine as the topsignaling pathway. Genes involved in oxidative stress response and apoptosis signaling pathway were also commonly upregulated (Supplementary Figure 8), which is consistent with the apoptotic cell death in these cells. We next perform a common pathway analysis using a Venn diagram between Day2 and other three DKO groups. Thirty pathways, including the apoptosis signaling pathway, were found to be commonly upregulated among IL-6-treated erythroblasts in vitro and DKO erythroblasts in vivo (Figure $6 \mathrm{~d}$ ). When these 30 pathways were further aligned by percentage of gene hits and number of hit genes, the inflammation pathway mediated by chemokine and cytokine was the most common pathway (Figure 6e), which further confirms that the ineffective erythropoiesis in DKO mice is directly related to the IL-6-enriched inflammatory bone marrow environment.

\section{DISCUSSION}

In this study, we reveal the significant roles of age-related inflammation in bone marrow microenvironment and its cooperative interaction with chromosome $5 q$ deleted genes in the pathogenesis of ineffective erythropoiesis in MDS. Aged bone marrow accumulates DAMPs and PAMPs, which direct activation and expansion of MDSCs, especially those primed by deletion of mDia1 and miR-146a, for excess IL-6 and TNFa elaboration. These proinflammatory cytokines accumulate to a pathologic level and trigger the over-production of reactive oxygen species that initiate caspase-mediated erythroid cell death, or GATA1 degradation leading to the inhibition of terminal erythropoiesis. The apoptotic erythroid cells further produce DAMPs, including S100 proteins, which creates a positive-feedback loop to enhance the inflammatory bone marrow microenvironment-mediated ineffective erythropoiesis (Figure 6f). 
a
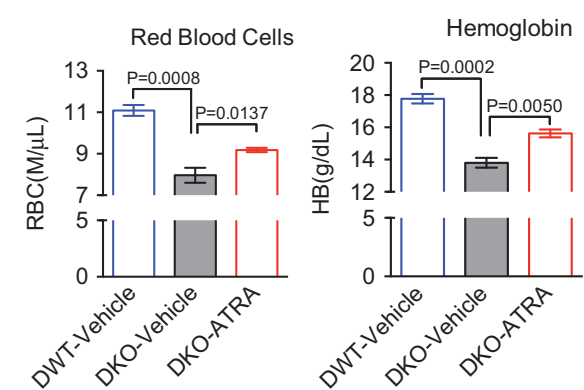

C

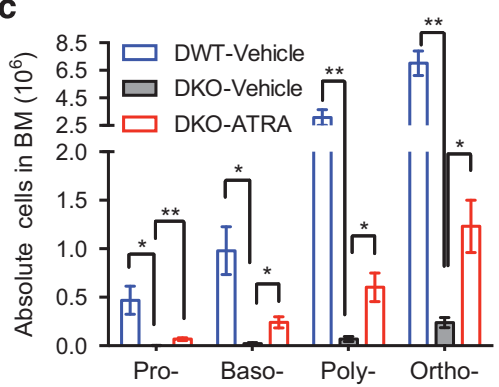

e

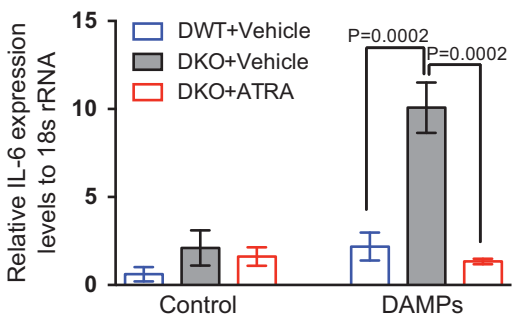

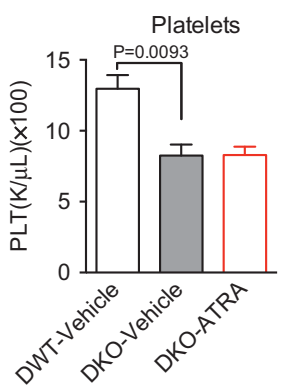

d
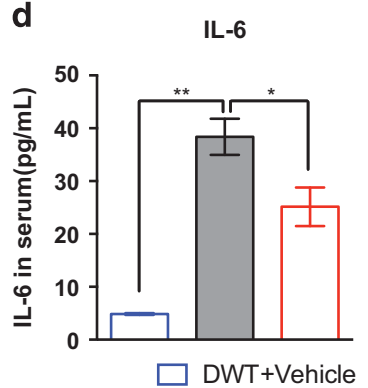

TNF $\propto$ mRNA

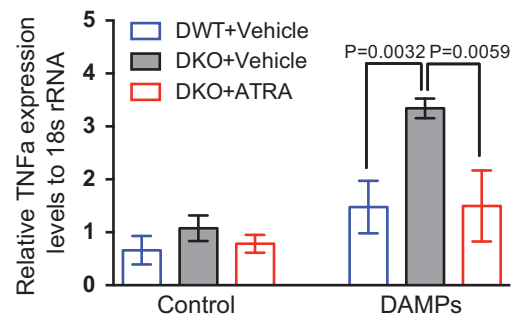

h

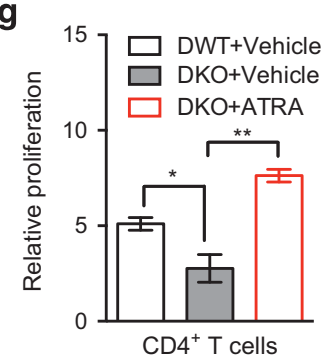

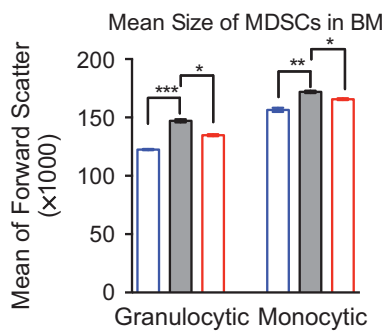

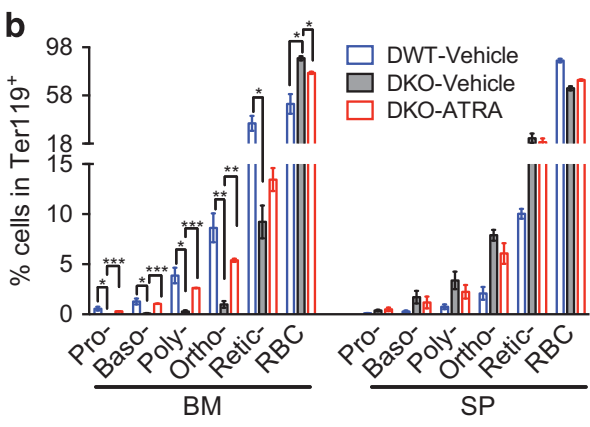

TNF $\alpha$

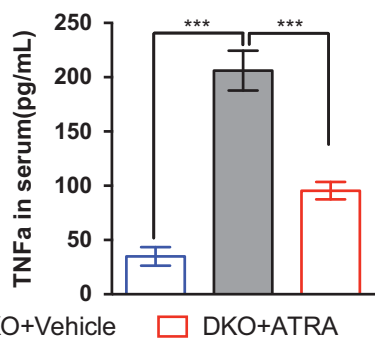

f

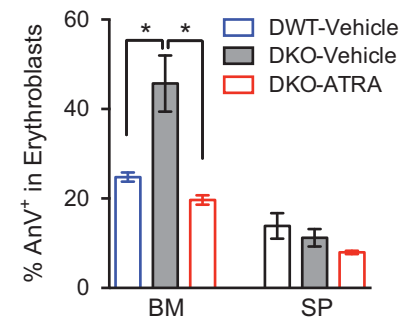

Figure 5. Amelioration of anemia in DKO mice by targeting MDSCs with AT-RA treatment. (a) DWT or DKO mice were intraperitoneally injected with $400 \mu \mathrm{g}$ ATRA dissolved in $100 \mu \mathrm{l}$ DMSO, or equal volume of vehicle control every two days for three doses. $48 \mathrm{~h}$ after the last injection, red blood cell counts and hemoglobin levels were assayed. (b, c). The percentages of erythroid cells at different stages were examined by flow cytometry in Ter119-positive cells from the bone marrow and spleen from mice in a. The absolute erythroblasts cell number was calculated in c. (d) Serum TNFa and IL-6 cytokine levels were determined in mice with or without ATRA treatment. (e) Gr1 and Mac1 double-positive MDSCs were purified from the indicated mice and challenged with 1:20 DAMPs for $2 \mathrm{~h}$ in vitro. The mRNA levels of TNFa and IL-6 were assayed by realtime PCR using $18 \mathrm{~s}$ rRNA as an internal control. (f) The apoptotic erythroblasts in the bone marrow and spleen from the indicated mice were analyzed by Annexin V and PI staining followed by flow cytometric analysis. (g) Gr1 and Mac1 double-positive MDSCs were purified and cocultured with cell proliferation dye labeled-splenic $T$ cells at 1:1 ratio, the mean fluorescence intensity (MFI) were determined by flow cytometric assay after $48 \mathrm{~h}$ stimulation by anti-CD3/CD28 coated Dynabeads. The proliferation rate was calculated as percentage decrease of

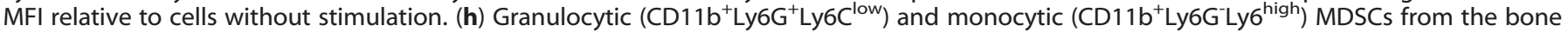
marrow and peripheral blood of mice in a were assayed by flow cytometry. $N=3-5$ mice in each group. ${ }^{*} P<0.05,{ }^{* *} P<0.01,{ }^{* * *} P<0.001$.

Deletions of $5 q$ genes in $\operatorname{del}(5 q)$ MDS are almost uniformly hemizygous. Although other genetic abnormalities in the retained $5 q$ alleles have not been detected so far, recent studies indicate that many genes on the retained $5 q$ alleles are epigenetically silenced. ${ }^{32,33}$ In fact, epigenetic dysregulation is commonly seen in MDS leading to the loss of function of many tumor suppressor proteins. $^{34}$ Therefore, we employed the mDia1 and miR-146a double knockout mouse model instead of the double heterozygous mice in this study. This approach also allows us to compare the current data with previous reports using miR-146 knockout mice to study myeloid neoplasms. ${ }^{35-37}$

The Gr1 and Mac1 double-positive myeloid cells in old DKO mice fulfill the criteria of MDSCs with their immature morphology and T cell suppressive activity. In our model, these MDSCs also potently suppress erythropoiesis. Therefore, the erythroid suppressive function represents a key pathologic feature of MDSCs as 
a

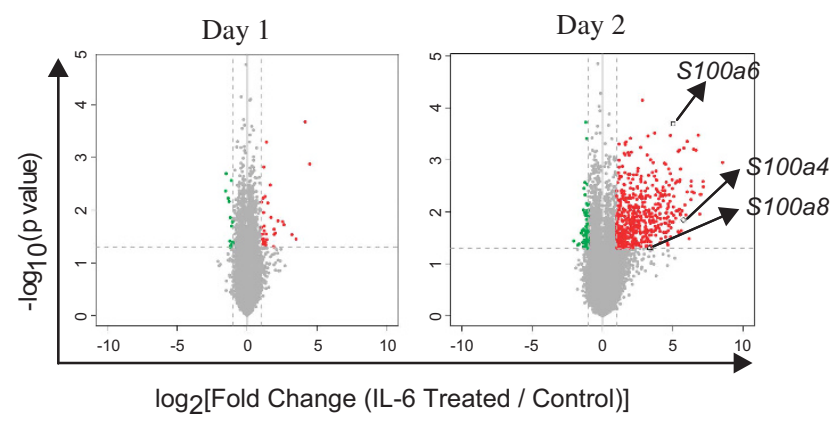

C

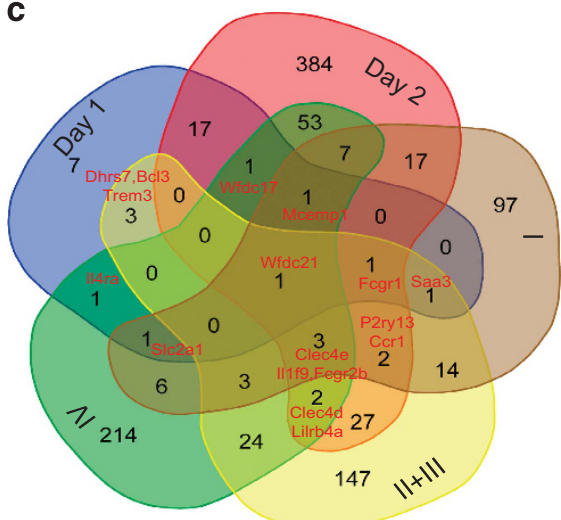

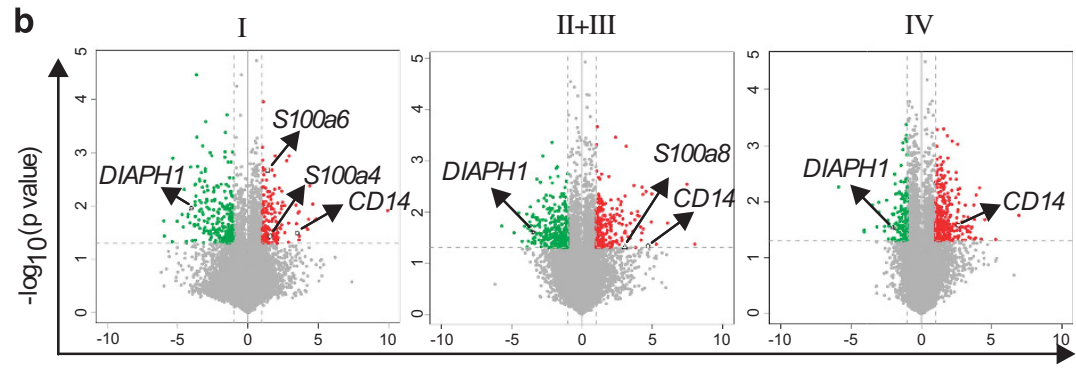

$\log _{2}$ [Fold Change (DKO / DWT)] d

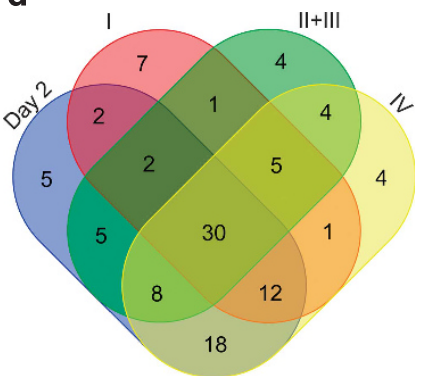

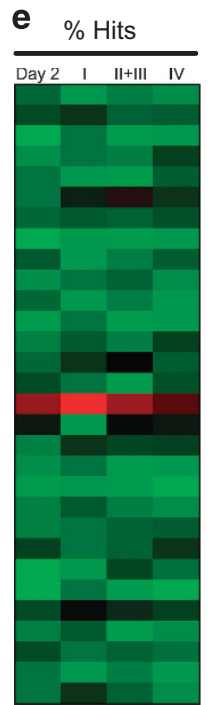

Min

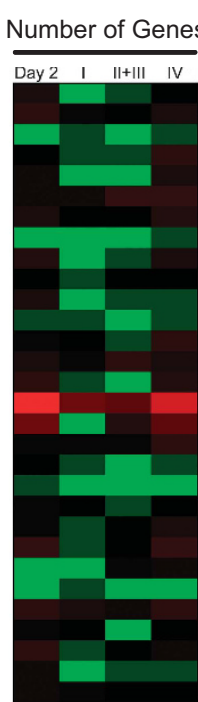

$\operatorname{Max}$
Pathways

Pathways

Angiogenesis (P00005)

Angiotensin II-stimulated signaling through $\mathrm{G}$ proteins and $\beta$-arrestin (P05911) Apoptosis signaling pathway (P00006)

B cell activation (P00010)

CCKR signaling map (P06959)

Cytoskeletal regulation by Rho GTPase (P00016)

De novo purine biosynthesis (P02738)

EGF receptor signaling pathway (P00018)

Endothelin signaling pathway (P00019)

FGF signaling pathway (P00021)

GABA-B receptor II signaling (P05731)

Gonadotropin-releasing hormone receptor pathway (P06664)

Gia and Gsa mediated pathway (P00026)

Huntington disease (P00029)

(P00031)

Integrin signalling pathway (P00034)

(

4 signaling pathway ( $\mathrm{P} 00043$ )

(P06587)

pathway (P00044)

arkinson disease (P00049)

GF signaling pathway (P00047)

PI3 kinase pathway (P00048)

alvage pyrimidine ribonucleotides ( $\mathrm{P} 02775$ )

cell activation (P00053)

GF-beta signaling pathway (P00052)

oll receptor signaling pathway (P00054)

GF signaling pathway (P00056) f

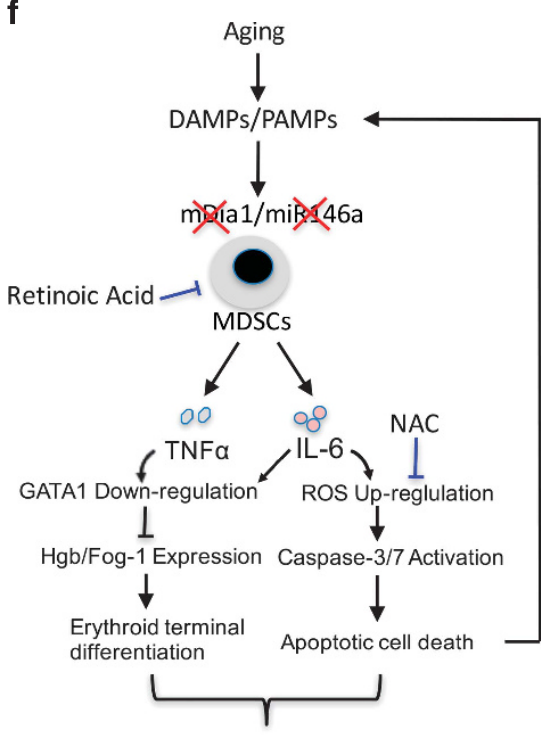

Ineffective Erythropoiesis

Figure 6. In vitro IL-6-treated bone marrow erythroblasts share the same gene expression profiles as the erythroblasts from DKO mice. (a, b) Volcano plot of microarray data, as generated by clustering based on the genes that were significantly enriched (red dots) or depleted (green dots) (fold change $>2$; ANOVA $P<0.05$ ) in IL-6-treated (a) or DKO erythroblasts (b) over their controls. Representative up or downregulated genes are pointed out. I represents proerythroblasts, II+III represent basophilic and polychromatic erythroblasts, IV represents orthochromatic erythroblasts. (c) Venn diagram defining significantly upregulated genes in in vitro IL-6-treated erythroblasts and erythroblasts from different developmental stages in DKO mice and compared with their corresponding controls. The numbers of overlapped genes are also shown. (d) Venn diagram showing the overlapping signaling pathways generated by PANTHER pathway analysis among the indicated groups (d). 30 signaling pathway were enriched both in Day 2 IL-6-treated erythroblasts and DKO erythroblasts. (e) The percentage of gene hits against total numbers of Pathway hits and gene numbers in specific pathway are illustrated by color scales. (f) Model of the age-related bone marrow microenvironment in the pathogenesis of ineffective erythropoiesis in del(5q) MDS.

previously reported. ${ }^{10,18,22}$ By targeting MDSCs through all-trans retinoic acid (ATRA), we found improved erythropoiesis in DKO mice. Mechanically, ATRA attenuated the cytokine upregulation both in vivo and in vitro, through the promotion of cell differentiation evidenced by the reduced cell size of MDSCs
post-ATRA treatment and the percentage of monocytic-MDSCs. In fact, several clinical trials using ATRA to treat MDS have been reported. ${ }^{38-40}$ One report showed ATRA combined with erythropoietin was effective for the treatment of anemia in low risk MDS patients having failed erythropoietin alone. ${ }^{38}$ Our study provides 
mechanistic insights showing that ATRA ameliorates anemia in MDS through reduction of MDSC-induced inflammatory bone marrow microenvironment.

TNFa and IL-6 are known to inhibit erythropoiesis. ${ }^{41}$ However, the detailed mechanisms of how different cytokines are involved in ineffective erythropoiesis are unclear. One of the most commonly involved pathways in inflammation-related anemia is cytokine-mediated hepcidin upregulation in the liver, which negatively affects the availability of iron to the developing erythroblasts. $^{25,42}$ However, hepcidin is not increased in our DKO mouse model. Instead, our study revealed direct but distinct roles of TNFa and IL- 6 in the pathogenesis of ineffective erythropoiesis. Both cytokines blocked erythroid colony formation. After the CFUE stage, IL- 6 induced potent upregulation of reactive oxygen species and caspase-mediated cell death whereas TNFa mainly affects GATA1 protein levels. These results are also consistent with reported clinical observations that IL- 6 and activated caspase- 1 and caspase- 3 are upregulated in MDS patients with refractory anemia. ${ }^{9,10}$

IL-6 is upregulated in miR-146a knockout mice through increased TRAF6, a well-known target of miR-146a. ${ }^{35}$ Our previous study shows that IL- 6 and TNFa are also prone to be upregulated in mDia1 deficient mice when the mice are subject to innate immune stimulation, related to increased expression of CD14 on granulocytes. ${ }^{5}$ Here we show that the combined deficiency of miR-146a and mDia1 leads to a profoundly increased elaboration of these cytokines, especially IL-6. This increase is most prominent in aged mice, which is consistent with the age-related changes in the bone marrow microenvironment. The level of IL- 6 correlates with the level of ineffective erythropoiesis. In miR-146a/mDia1 double knockout mice, IL- 6 is nearly four-fold higher than miR-146a knockout mice and 20-30-fold higher than the wild type or mDia1 knockout mice. Correspondingly, bone marrow erythroid cells are significantly reduced in miR-146a knockout mice and almost completely absent in miR-146a/mDia1 double knockout mice. These results are consistent with a more severe effect of IL-6 in erythropoiesis. Overall, our study highlights the significance of the bone marrow inflammatory microenvironment in the pathogenesis of ineffective erythropoiesis and anemia in del (5q) MDS.

\section{CONFLICT OF INTEREST}

The authors declare no conflict of interest.

\section{ACKNOWLEDGEMENTS}

We thank Matthew Schipma for the help with the analysis of gene expression profiles. Dr. Lin Li at the Mouse Phenotyping Facility of the Northwestern University for the help with the mouse histology. The work is supported by a National Institute of Diabetes and Digestive and Kidney Disease (NIDDK) grant DK102718 (PJ), Department of Defense grant CA140119 (PJ).

\section{REFERENCES}

1 Ebert BL. Molecular dissection of the $5 q$ deletion in myelodysplastic syndrome. Semin Oncol 2011; 38: 621-626.

2 Pellagatti A, Boultwood J. Recent Advances in the 5q-Syndrome. Mediterr J Hematol Infect Dis 2015; 7: e2015037.

3 Lefèvre C, Bondu S, Le Goff S, Kosmider O, Fontenay M. Dyserythropoiesis of myelodysplastic syndromes. Curr Opin Hematol 2017; 24: 191-197.

4 Schneider RK, Schenone M, Ferreira MV, Kramann R, Joyce CE, Hartigan C et al. Rps14 haploinsufficiency causes a block in erythroid differentiation mediated by S100A8 and S100A9. Nat Med 2016; 22: 288-297.

5 Keerthivasan G, Mei Y, Zhao B, Zhang L, Harris CE, Gao J et al. Aberrant overexpression of CD14 on granulocytes sensitizes the innate immune response in mDia1 heterozygous del(5q) MDS. Blood 2014; 124: 780-790.

6 Fang J, Bolanos LC, Choi K, Liu X, Christie S, Akunuru S et al. Ubiquitination of hnRNPA1 by TRAF6 links chronic innate immune signaling with myelodysplasia. Nat Immunol 2017; 18: 236-245.
7 Varney ME, Niederkorn M, Konno H, Matsumura T, Gohda J, Yoshida N et al. Loss of Tifab, a del(5q) MDS gene, alters hematopoiesis through derepression of Tolllike receptor-TRAF6 signaling. J Exp Med 2015; 212: 1967-1985.

8 Komrokji RS, Kulasekararaj A, Ali Al NH, Kordasti S, Bart-Smith E, Craig BM et al. Autoimmune diseases and myelodysplastic syndromes. Am J Hematol 2016; 91 : E280-E283.

9 Basiorka AA, McGraw KL, Eksioglu EA, Chen X, Johnson J, Zhang L et al. The NLRP3 inflammasome functions as a driver of the myelodysplastic syndrome phenotype. Blood 2016; 128: 2960-2975.

10 Lambert C, Wu Y, Aanei C. Bone Marrow Immunity and Myelodysplasia. Front Oncol 2016; 6: 172

11 Starczynowski DT, Kuchenbauer F, Argiropoulos B, Sung S, Morin R, Muranyi A et al. Identification of miR-145 and miR-146a as mediators of the $5 q$ - syndrome phenotype. Nat Med 2010; 16: 49-58.

12 Boldin MP, Taganov KD, Rao DS, Yang L, Zhao JL, Kalwani M et al. miR-146a is a significant brake on autoimmunity, myeloproliferation, and cancer in mice. $J$ Exp Med 2011; 208: 1189-1201.

13 Liu J, Zhang J, Ginzburg Y, Li H, Xue F, De Franceschi L et al. Quantitative analysis of murine terminal erythroid differentiation in vivo: novel method to study normal and disordered erythropoiesis. Blood 2013; 121: e43-e49.

14 An X, Schulz VP, Mohandas N, Gallagher PG. Human and murine erythropoiesis. Curr Opin Hematol 2015; 22: 206-211.

15 Perry JM, Harandi OF, Paulson RF. BMP4, SCF, and hypoxia cooperatively regulate the expansion of murine stress erythroid progenitors. Blood 2007; 109: 4494-4502.

16 Porayette P, Paulson RF. BMP4/Smad5 dependent stress erythropoiesis is required for the expansion of erythroid progenitors during fetal development. Dev Biol 2008; 317: 24-35.

17 Millot S, Andrieu V, Letteron P, Lyoumi S, Hurtado-Nedelec M, Karim Z et al. Erythropoietin stimulates spleen BMP4-dependent stress erythropoiesis and partially corrects anemia in a mouse model of generalized inflammation. Blood 2010; 116: 6072-6081.

18 Chen X, Eksioglu EA, Zhou J, Zhang L, Djeu J, Fortenbery $\mathrm{N}$ et al. Induction of myelodysplasia by myeloid-derived suppressor cells. J Clin Invest 2013; 123: 4595-4611.

19 Movahedi K, Guilliams M, Van den Bossche J, Van den Bergh R, Gysemans C, Beschin $\mathrm{A}$ et al. Identification of discrete tumor-induced myeloid-derived suppressor cell subpopulations with distinct T cell-suppressive activity. Blood 2008; 111: 4233-4244.

20 Huang J, Xie Y, Sun X, Zeh HJ, Kang R, Lotze MT et al. DAMPs, ageing, and cancer: The 'DAMP Hypothesis. Ageing Res Rev 2015; 24: 3-16.

21 Lotfi R, Herzog Gl, DeMarco RA, Beer-Stolz D, Lee JJ, Rubartelli A et al. Eosinophils oxidize damage-associated molecular pattern molecules derived from stressed cells. J Immunol 2009; 183: 5023-5031.

22 Gabrilovich DI, Nagaraj S. Myeloid-derived suppressor cells as regulators of the immune system. Nat Rev Immunol 2009; 9: 162-174.

23 Gardenghi S, Renaud TM, Meloni A, Casu C, Crielaard BJ, Bystrom LM et al. Distinct roles for hepcidin and interleukin- 6 in the recovery from anemia in mice injected with heat-killed Brucella abortus. Blood 2014; 123: 1137-1145.

24 Lee $\mathrm{P}$, Peng H, Gelbart T, Wang L, Beutler E. Regulation of hepcidin transcription by interleukin-1 and interleukin-6. Proc Natl Acad Sci USA 2005; 102: 1906-1910.

25 Andrews NC. Anemia of inflammation: the cytokine-hepcidin link. J Clin Invest 2004; 113: 1251-1253.

26 Crispino JD. GATA1 in normal and malignant hematopoiesis. Semin Cell Dev Biol 2005; 16: 137-147.

27 Circu ML, Aw TY. Reactive oxygen species, cellular redox systems, and apoptosis. Free Radic Biol Med 2010; 48: 749-762.

28 Zhao B, Mei Y, Yang J, Ji P. Erythropoietin-regulated oxidative stress negatively affects enucleation during terminal erythropoiesis. Exp Hematol 2016; 44: 975-981.

29 Nefedova Y, Fishman M, Sherman S, Wang X, Beg AA, Gabrilovich DI. Mechanism of all-trans retinoic acid effect on tumor-associated myeloid-derived suppressor cells. Cancer Res 2007; 67: 11021-11028.

30 Long AH, Highfill SL, Cui Y, Smith JP, Walker AJ, Ramakrishna S et al. Reduction of MDSCs with All-trans Retinoic Acid Improves CAR Therapy Efficacy for Sarcomas. Cancer Immunol Res 2016; 4: 869-880.

$31 \mathrm{Mi} \mathrm{H}$, Muruganujan A, Casagrande JT, Thomas PD. Large-scale gene function analysis with the PANTHER classification system. Nat Protoc 2013; 8: 1551-1566.

32 Liu TX, Becker MW, Jelinek J, Wu W-S, Deng M, Mikhalkevich N et al. Chromosome $5 q$ deletion and epigenetic suppression of the gene encoding a-catenin (CTNNA1) in myeloid cell transformation. Nat Med 2006; 13: 78-83.

33 Ye Y, McDevitt MA, Guo M, Zhang W, Galm O, Gore SD et al. Progressive chromatin repression and promoter methylation of CTNNA1 associated with advanced myeloid malignancies. Cancer Res 2009; 69: 8482-8490.

34 Khan $\mathrm{H}$, Vale $\mathrm{C}$, Bhagat $\mathrm{T}$, Verma A. Role of DNA methylation in the pathogenesis and treatment of myelodysplastic syndromes. Semin Hematol 2013; 50: 16-37. 
35 Lu L-F, Boldin MP, Chaudhry A, Lin L-L, Taganov KD, Hanada T et al. Function of miR-146a in controlling Treg cell-mediated regulation of Th1 responses. Cell 2010; 142: 914-929.

36 Zhao JL, Rao DS, O'Connell RM, Garcia-Flores Y, Baltimore D. MicroRNA-146a acts as a guardian of the quality and longevity of hematopoietic stem cells in mice. Elife 2013; 2: e00537.

37 Yang L, Boldin MP, Yu Y, Liu CS, Ea C-K, Ramakrishnan P et al. miR-146a controls the resolution of T cell responses in mice. J Exp Med 2012; 209: 1655-1670.

38 Itzykson R, Ayari S, Vassilief D, Berger E, Slama B, Vey N et al. Is there a role for alltrans retinoic acid in combination with recombinant erythropoetin in myelodysplastic syndromes? A report on 59 cases. Leukemia 2009; 23: 673-678.

39 Baldus M, Walter H, Möller M, Schürfeld C, Brass H. ALL-trans retinoic acid (ATRA) in the treatment of myelodysplastic syndromes: results in 5 cases. Oncol Res Treat 2004; 17: 515-520.

40 Stasi R, Brunetti M, Terzoli E, Amadori S. Sustained response to recombinant human erythropoietin and intermittent all-trans retinoic acid in patients with myelodysplastic syndromes. Blood 2002; 99: 1578-1584.
41 Morceau F, Dicato M, Diederich M. Pro-inflammatory cytokine-mediated anemia: regarding molecular mechanisms of erythropoiesis. Mediators Inflamm 2009; 2009: 405016-11.

42 D'Angelo $G$. Role of hepcidin in the pathophysiology and diagnosis of anemia. Blood Res 2013; 48: 10-15.

This work is licensed under a Creative Commons Attributioncc) NonCommercial-ShareAlike 4.0 International License. The images or other third party material in this article are included in the article's Creative Commons license, unless indicated otherwise in the credit line; if the material is not included under the Creative Commons license, users will need to obtain permission from the license holder to reproduce the material. To view a copy of this license, visit http:// creativecommons.org/licenses/by-nc-sa/4.0/

(c) The Author(s) 2018

Supplementary Information accompanies this paper on the Leukemia website (http://www.nature.com/leu) 